\title{
MEJORA DE LOS PROCESOS EN UNA EMPRESA COMERCIALIZADORA DE ARTÍCULOS DE FERRETERÍA USANDO TÉCNICAS DE ANÁLISIS DE VALOR AGREGADO Y UN MODELO Y NOTACIÓN DE PROCESOS DE NEGOCIOS
}

\author{
IMPROVE OF THE PROCESS IN A COMPANY TRADING OF HARDWARE STORE \\ ARTICLESUSING TECHNICAL OF ANALYSIS VALUE ADDED AND BUSINESS \\ PROCESS MANAGEMENT NOTE
}

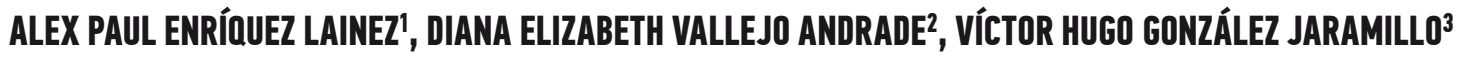

\footnotetext{
1 Escuela Superior Politécnica del Litoral. alepenri@espol.edu.ec

2 Escuela Superior Politécnica del Litoral. devallej@espol.edu.ec

3 Escuela Superior Politécnica del Litoral. vgonzal@espol.edu.ec
}

\begin{abstract}
Se analizaron los desperdicios de los procesos de una pequeña empresa familiar, comercializadora de productos de ferretería. Se realizó un levantamiento de procesos actuales, para luego elaborar un análisis de valor agregado a los mismos, construyendo indicadores de control y análisis de valor para determinar los desperdicios. Como metodología, se utilizaron cuestionarios y mediciones en la empresa, identificando los procesos actuales, y elaborando los nuevos, usando la técnica de Modelo y Notación de Procesos de Negocios (BPMN) con el software Bizagi ${ }^{\circledR}$. Se determinó que las áreas más conflictivas de la empresa son las administrativas de servicio al cliente, y bodega. Usando la misma técnica anterior, BPMN, se re-diseñaron los procesos futuros, de compra y recepción de mercadería, servicio y atención al cliente, obteniendo una mejora global del $17 \%$. Con este avance positivo, se le dio a la empresa un manual de procesos con los indicadores de control, con el fin de que se pueda aplicar esta metodología.
\end{abstract}

PALABRAS CLAVE: análisis de valor agregado, notación de procesos de negocios.
ABSTRACT

In this applied research, waste processes of a family small business marketer of hardware were analyzed. A survey of current processes were performed to produce a value analysis to design indicators to locate the waste. These questionnaires and measurements were used in the company to identify the current processes, and develop new processes using (Business Process Management Note (BPMN) with the software Bizagi ${ }^{\circledR}$. It was found that the most troubled areas of the business were the administrative areas of customer service, and warehouse. Using the same technique, BPMN, a re-design was performed of the future processes, of purchasing goods, receiving goods, and customer service, obtaining an overall improvement of $17 \%$. Finally, a process manual with control indicators was given to the company to enhance future improvements.

KEYWORDS: value-added analysis, Business Process Management Note. 
INTRODUCCIÓN

Teniendo una reunión con los dueños del establecimiento se pudo notar que tienen algunos inconvenientes con la organización de los procesos, lo cual a futuro puede ocasionar problemas a nivel interno y reflejarse en inconsistencias a la hora de atender al cliente. Como la empresa en la actualidad no está completamente organizada en cuanto a sus procesos, lo que más afecta es la falta de control en su inventario porque perjudica bastante al comprador ya que se prolonga su tiempo de espera, haciendo que éste se desespere y concluya que la atención al cliente no cumple con sus expectativas perjudicando a su vez las ventas del negocio.

Con este estudio se desea además de implementar mejoras en sus procesos, elaborar indicadores de control que sirvan como parámetros para diseñar e implementar los nuevos procesos para el servicio al cliente, compra y recepción de mercadería, en donde se evalúan estos procesos, identificando los factores que afectan al rendimiento de la empresa y de esta forma optimizar el funcionamiento de la misma y escoger las estrategias más oportunas para cada caso, que ayuden en la gestión apropiada en cuanto a la forma de administrar la compañía, elevando la confiabilidad, la eficiencia, eficacia y garantizando un servicio de calidad.

\section{MATERIALES Y MÉTODOS} INDICADORES DE CONTROL

Un indicador de control es aquel que es claro, relevante, adecuado, monitoreable, económico y que brinde un aporte marginal, para que no sea mal interpretado y no exista ningún tipo de equivocación; relevante, es decir, relacionados con los objetivos, para saber si se están cumpliendo o no con la meta del indicador; adecuado, con respecto a emitir una información que sea relevante y apropiada en el que se pueda describir las metas alcanzadas del programa en un determinado tiempo; monitoreable, para analizar los medios de verificación que deben ser precisos; económico, en el cual además del costo económico se evalúa el costo humano para la validación; y que brinde un aporte marginal referido a lograr un buen desempeño y cumplir con sus objetivos para el éxito del proceso (CONEVAL, 2013). Se debe tener presente los criterios de valoración para que estos indicadores se diseñen, teniendo en cuenta las principales características que se mencionaron anteriormente (Público, 2010).
ANÁLISIS DEL VALOR AGREGADO

Este análisis es una herramienta esencial para verificar que un proceso sea eficiente. Cuando un proceso cumple con el valor agregado quiere decir que satisface las necesidades del cliente. El análisis del valor agregado se divide en:

Actividades de no valor agregado:

1. Valor agregado para el cliente (VAC)

2. Valor agregado para la empresa (VAE)

Actividades de valor agregado:

1. Preparación $(\mathrm{P})$

2. Inspección (I)

3. Espera (E)

4. Movimiento(M)

5. Archivo (A)

El motivo principal de este análisis fue identificar las actividades de no valor y eliminarlas con el fin de mejorar el resto de actividades (Moreira, 2009). Una vez identificadas las tareas que agregan valor, se proceden a elaborar diagramas de flujo con BPMN, estableciendo tiempos por cada actividad para luego calcular el índice del valor agregado y mejorar los procesos mediante la simulación.

\section{BPMN \& IDEF (INTEGRATION DEFINITION FOR FUNCTION} MODELING)

BPMN sirve para representar la notación y el modelo de procesos de negocios, y el software que se utilizó para este fin fue el Bizagi ${ }^{\circledR}$, ya que nos ayudó a simular los procesos y las interacciones existentes en el modelo de negocios (Bizagi, s.f.). Las variables que integran estos modelos fueron determinadas por medio de cuestionarios con el fin de diagnosticar en donde ocurren los problemas de cada proceso. IDEF sirve para organizar la información y diagramar de manera jerárquica y estructurada, cada uno de los procesos fundamentales que sustentan la organización (Informáticas, 2012).

\section{DOCUMENTACIÓN DE PROCESOS}

Esta actividad se debe realizar básicamente para una mayor comprensión de los procesos de la organización, los cuales deben de ser registrados con el fin de determinar ¿Qué?, ¿Cómo? y ¿Por qué? ha sucedido algún evento y de esta forma llegar a identificar cuáles son las fallas que podrían estar ocasionando problemas en la organización (Schouten, 2007).

"De muchas formas, la documentación del proceso de un movimiento de cambio social 
cumple la misma función que el diario de la vida de una persona. Un diario permite la reflexión diaria sobre eventos y los factores que enmarcan dichos eventos además de crear una especie de registro histórico. Con el tiempo, un diario, al igual que la documentación de procesos, revelará temas y patrones recurrentes que favorecen o dificultan el progreso hacia una transformación” (E. Casey, 2003, p.3).

La documentación de procesos se relaciona con la vida real, mejorando la situación y cambiando su cultura, patrones de poder, e incluso la toma de decisiones que se ha estado siguiendo dentro de la empresa (Schouten, 2007). Antes de la documentación de procesos, se debe tener en cuenta que un proceso tiene a su vez una serie de actividades o de subprocesos, por tal motivo los procesos son considerados como una cadena de valor (Servicios, s.f.).

Una vez que los procesos fueron bien identificados y puestos en su respectivo manual se procedió a su correcta documentación, siguiendo las siguientes etapas:

1. Definir el plan de acción para documentar procesos.

2. Definir un equipo de trabajo temático.

3. Analizar, evaluar y diagnosticar los procesos.

4. Rediseñar los procesos (Métodos, 2013).

ANÁLISIS DE RESULTADOS

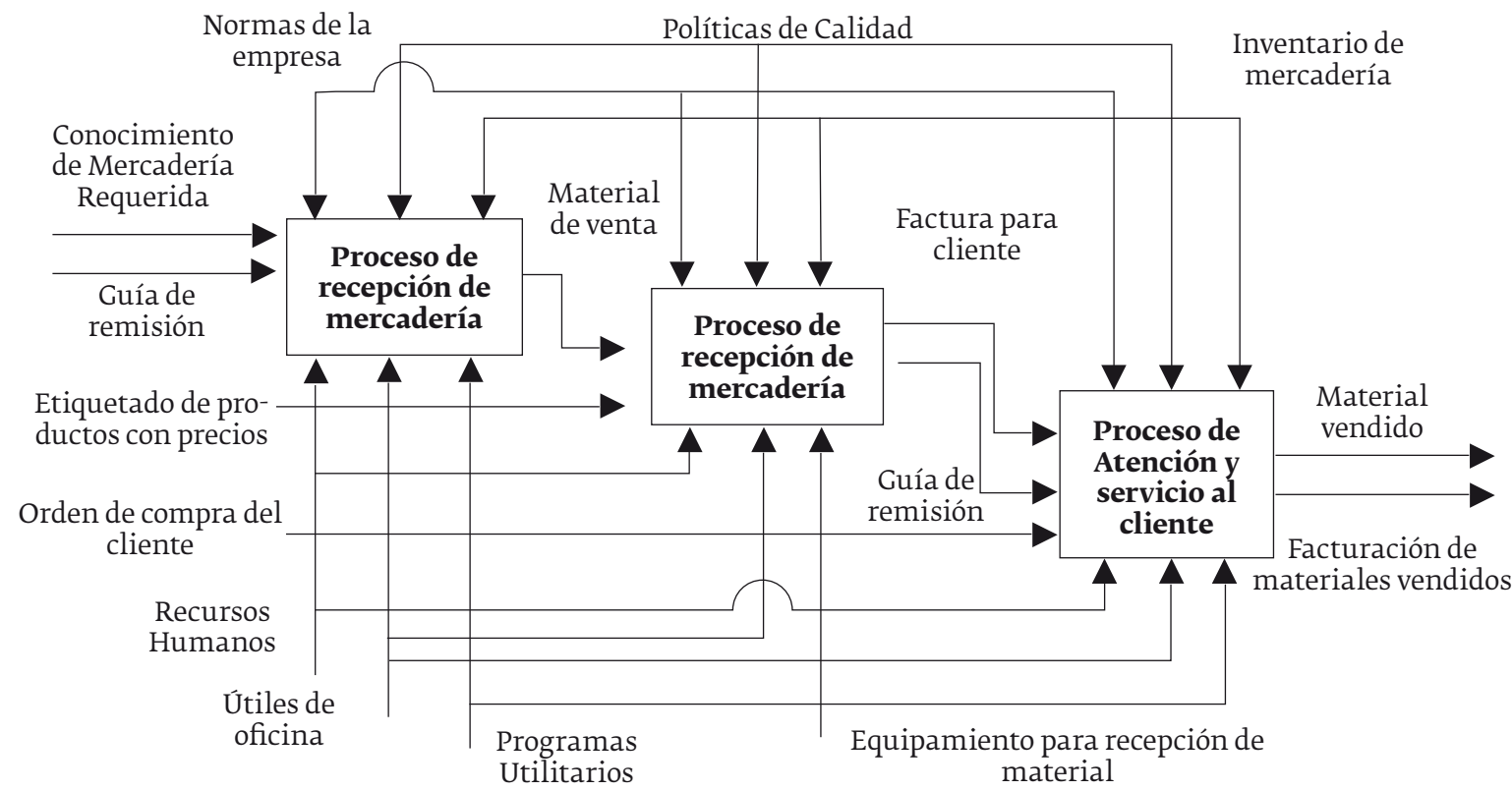

Figura 1. Mapa de procesos.

Fuente: IDEFO (Tributaria, 2011)
Los procesos que se verán a continuación fueron desarrollados según las actividades que se llevaban a cabo en las diferentes áreas de la empresa. De aquí, se determinaron los problemas mediante la metodología mencionada y se procedió a implementar las mejoras para que las actividades de la empresa sean óptimas.

\section{DETERMINACIÓN Y ELIMINACIÓN DE PROBLEMAS Y DESPERDICIOS}

Para determinar cuáles eran los desperdicios dentro de la empresa, en primera instancia se los localizó a través de la recolección de información mediante cuestionarios en los que se dio a conocer, por los mismos empleados, los procesos que se utilizan teniendo un diseño más preciso con la técnicas IDEF0 (Tributaria, 2011) y Bizagi ${ }^{\circledR}$. A continuación podemos observar en la figura 1 el diseño de los resultados obtenidos de cuestionarios en los cuales se procedió a analizar los escenarios actuales y futuros de los procesos críticos de la empresa.

Luego se procedió a indicar y detallar los procesos claves de la compañía, estos procesos fueron revisados por el Gerente y también se realizó un análisis en el que se determinó los procesos de mayor importancia y que causan un gran impacto dentro de la empresa.

Proceso de compra: esta actividad es primordial ya que cuando se detecta faltantes de productos, es necesario reabastecerse para poder cumplir con la demanda; se lleva a cabo una gestión de reconocimiento de qué productos se 
necesitan, y se realiza una lista documentada para hacer el pedido al proveedor respectivo.

Proceso de recepción de materiales: en el mismo proceso es necesario que se trabaje con claridad ya que se necesita que los productos recién llegados estén inmediatamente listos para la venta, es decir etiquetados con los respectivos precios y ubicados correctamente en los lugares designados.

Proceso de atención y servicio al cliente: es el más importante; mediante éste, se recepta la orden del cliente y es primordial que éste sea atendido en el menor tiempo posible y a su vez se trate de complacer en su requerimiento sin problemas ni fallos a la hora de la entrega del producto.

\section{COMPARACIÓN Y ANÁLISIS DE RESULTADOS ENTRE} PROCESOS ACTUALES Y FUTUROS

Al utilizar el método BPMN y el análisis de valor agregado, se establecieron las actividades que para la compañía no representan valor agregado en los procesos y por lo tanto generan demoras; se detectó que no había eficiencia en algunas actividades; para tratar de mejorar y optimizar el tiempo de las actividades se elaboró el manual de procesos y el mapa del macroproceso; con esta técnica se pueden optimizar los tiempos, y se estandarizó ciertas tareas para que sea más fácil realizarlas. Se puede visualizar en las figuras 2 y 3 los procesos de compra de mercadería, y de atención y servicio al cliente respectivamente.

El proceso de compra de mercadería es necesario para identificar los ítems faltantes, y proceder a realizar el pedido al proveedor, una vez llegada la mercadería se ubica el material nuevo en el sistema para poder contar inmediatamente con estos productos y ofrecerlos a los clientes; se deben ubicar correctamente en las áreas asignadas en bodega.

El proceso de atención y servicio al cliente de la figura 3 es importante para conocer cuáles son los inconvenientes al momento de atender a los compradores, y en qué partes existen las demoras que perjudican al proceso; así mismo las partes que intervienen son el vendedor, el encargado de bodega y el cajero; la empresa usa un procedimiento en la que se elabora un pedido que debe ser revisado por el asistente de bodega para determinar si se encuentra en stock, dependiendo de esto, se procede a realizar la factura, y no se despacha la mercadería hasta que no se haya cancelado en caja.

Los procesos tienen indicadores de control que inspecciona de una manera más rigurosa las actividades que se detallan a continuación.

Los indicadores del proceso de compra son: la duración del inventario, que busca que no exis$\tan$ faltantes de productos, la frecuencia con la que se debe utilizar (mensualmente); y la revisión de requisición de mercadería, que muestra

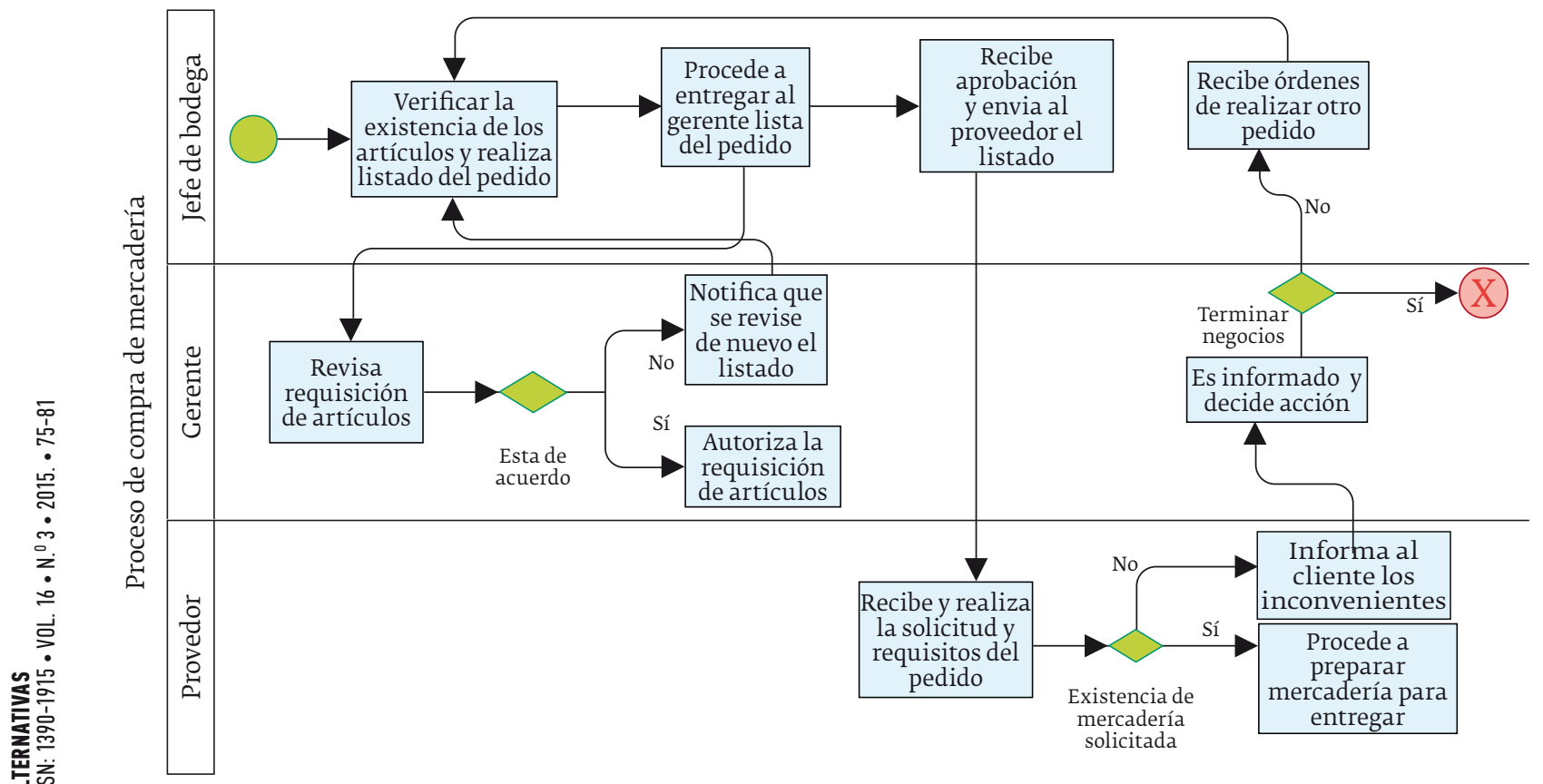

Figura 2. Proceso de compra de mercadería Fuente: los autores. 
con exactitud qué cantidad de inventario se necesitaría, de igual manera se debe revisar de forma mensual. En el proceso de recepción, se tiene como indicador la confiabilidad de la mercadería, se busca tener inventario sin errores, el porcentaje de infraestructura física y tecnológica que se ha implementado para la mejora; éstos se miden de forma mensual y trimestral respectivamente.

En el proceso de atención y servicio al cliente, el porcentaje de satisfacción busca mejorar el nivel de agrado del cliente para poder determinar si han sido eficientes, se controla de manera anual; el otro indicador es el de reducción de tiempo del proceso de despacho de mercadería, que busca optimizar el tiempo en el que se atiende y entrega el producto al comprador, éstos se evalúan de manera trimestral.

Una vez modelado el proceso se procedió a utilizar entrevistas y encuestas con la finalidad de medir los tiempos que requieren estas actividades; se implementó la técnica de valor agregado que nos permitió conocer donde se podía implementar la mejora. A continuación, en la tabla 1 , se realiza una comparación entre los

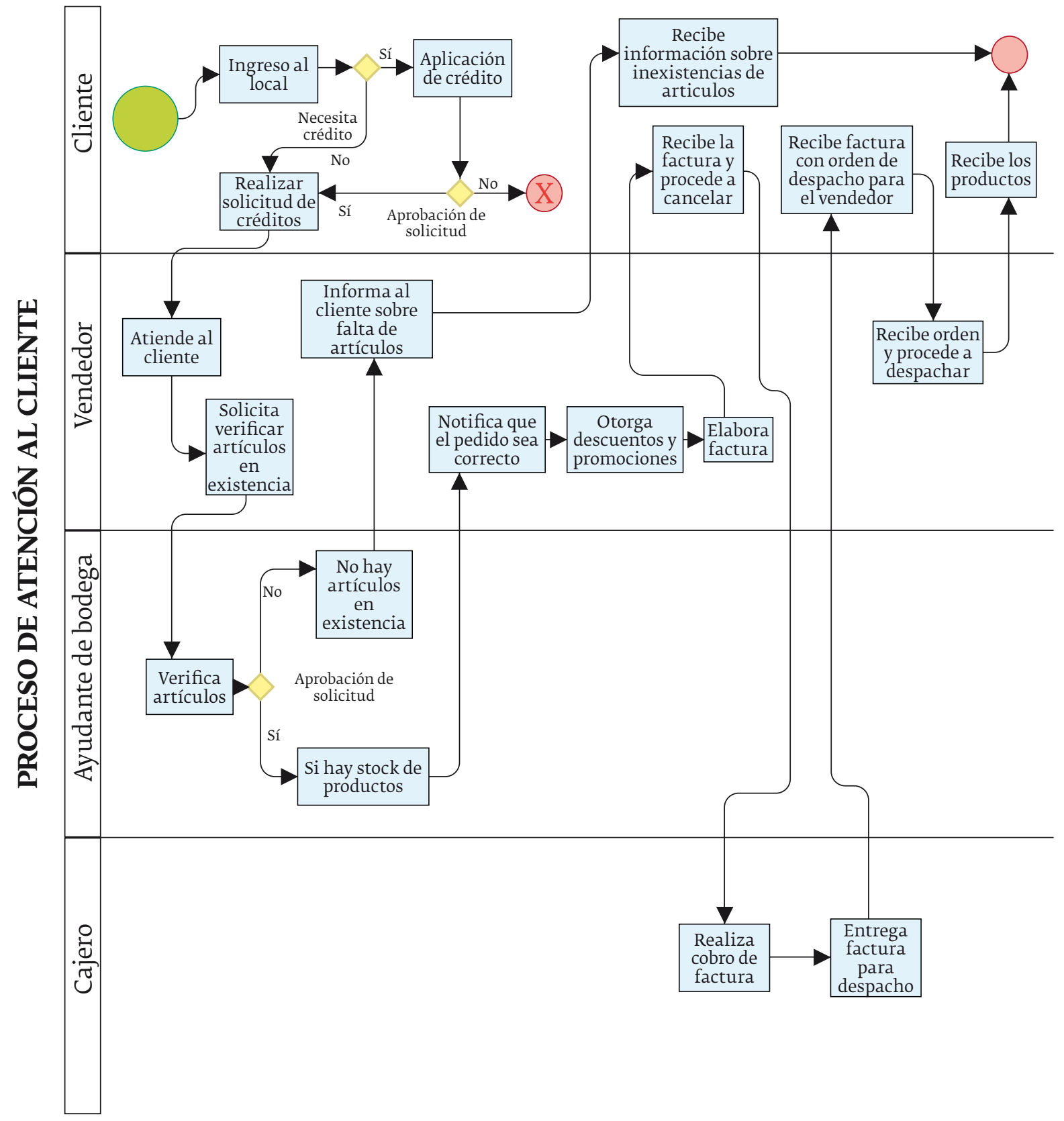

Figura 3. Proceso de atención y servicio al cliente. 


\begin{tabular}{|c|c|c|c|c|c|}
\hline \multicolumn{3}{|l|}{ 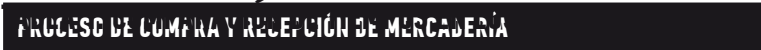 } & \multicolumn{3}{|l|}{ 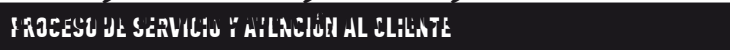 } \\
\hline \multirow[t]{2}{*}{ Verifica la existencia de los artículos y realiza pedido } & \multicolumn{2}{|c|}{ Tiempo (Minutos) } & \multirow[t]{2}{*}{ Estructura de Actividades } & \multicolumn{2}{|c|}{ Tiempo (Minutos) } \\
\hline & Actual & Futuro & & Actual & Futuro \\
\hline Verifica la existencia de los artículos y realiza pedido & 30 & 20 & Ingreso al local & vv & 1 \\
\hline Entrega a Gerente lista de pedido & 6 & 2 & Aplicación de crédito & 30 & 25 \\
\hline Revisa requisición de artículos & 15 & 10 & Realiza solicitud de artículos & 10 & 5 \\
\hline Notifica que se revise de nuevo el listado & 3 & 1 & Atiende al cliente & 8 & 6 \\
\hline Autoriza la requisición de artículos & 2 & 1 & Solicita verificar artículos en existencia & 2 & 1 \\
\hline Recibe aprobación y envía al proveedor el listado & 2 & 2 & Verifica artículos & 10 & 7 \\
\hline Recibe y realiza la solicitud y requisitos del pedido & - & - & No hay artículos en existencia & 2 & 2 \\
\hline Procede a preparar mercadería para entrega & - & - & Informa al cliente sobre falta de artículos & 1 & 1 \\
\hline Informa al cliente inconvenientes & - & - & Recibe información sobre inexistencias & 1 & 1 \\
\hline Es informado y decide acción & 15 & 10 & Si hay stock de productos & 2 & 1 \\
\hline Recibe órdenes de realizar otro pedido & 10 & 5 & Notifica que el pedido sea correcto & 5 & 3 \\
\hline Procede a realizar entrega de pedido & 20 & 10 & Otorga descuentos y promociones & 2 & 1 \\
\hline Entrega factura a jefe de bodega & 5 & 3 & Elabora factura & 10 & 8 \\
\hline Recibe factura & 3 & 1 & Recibe factura y procede a cancelar & 5 & 5 \\
\hline Descarga mercadería & 15 & 10 & Realiza cobro de factura & 5 & 2 \\
\hline Revisa que la mercadería esté completa & 5 & 5 & Entrega factura para despacho & 2 & 1 \\
\hline Informa que todo esté en orden & 5 & 1 & Recibe factura con orden de despacho & 1 & 1 \\
\hline Procede a entregar factura al gerente & 1 & 1 & Procede a despachar & 5 & 4 \\
\hline Recibe y analiza factura & 5 & 5 & Recibe productos & 2 & 1 \\
\hline Procede a etiquetar los productos & 25 & 20 & & & \\
\hline Procede a perchar mercadería & 30 & 25 & & & \\
\hline Tiempo Total & 197 & 132 & Tiempo Total & 105 & 76 \\
\hline
\end{tabular}

Fuente: los autores.

tiempos actuales y futuros del proceso de compra y recepción de mercadería, y el de servicio y atención al cliente.

Una vez realizado este estudio, se puede observar que con el rediseño se obtuvo una mejora en los tiempos. Con el análisis del valor agregado, se determinó que en el proceso de compra de mercadería, las actividades de valor agregado y sin valor agregado, cambian de un $39 \%$ al $43 \%$ (VA) y de un $61 \%$ al $57 \%$ (SVA). En el proceso de recepción la reducción fue desde un $48 \%$ al $56 \%$ (VA) y de un $52 \%$ a un $54 \%$ (SVA). En el proceso de servicio al cliente aumento el valor agregado (VA) del 61\% al 66\% y una disminución en el (SVA) de $39 \%$ a un $34 \%$, reduciendo de esta manera el tiempo de espera del cliente y mejorando los procesos para que sean más eficaces.
CONCLUSIONES

Luego de realizar el trabajo para esta empresa se pudo apreciar que el principal desperdicio que genera la compañía es la falta de supervisión en los procesos desde la llegada de mercadería hasta el servicio al cliente, ya que los problemas se detectan al momento de buscar la mercadería que solicita el cliente. Este inconveniente resultaba molesto para el comprador en razon de que deben esperar demasiado tiempo, y además suelen irse sin sus productos deseados debido a que en ese momento los empleados se enteran de las inexistencias. Por esto, se ha estandarizado los procesos con el manual de procesos entregado, de tal manera que se disminuyan los tiempos requeridos en las actividades.

Adicionalmente, se espera que los empleados sean instruidos con los procesos diseñados para 
que al final de cuentas se muestre una mejora de servicio percibido por el cliente. En lo posterior, es recomendable un seguimiento de la

\section{REFERENCIAS BIBLIOGRÁFICAS}

Bizagi. (s.f.). Recuperado el 15 de 08 de 2015, Obtenido de http://wiki.bizagi.com/es/index. php?title=BPMN

CONEVAL. (2013). Manual para el Diseño y la Construcción de Indicadores. Instrumentos principales para el monitoreo de programas sociales de México. México: Primera edición.

E. Casey. (2003). Accedido el 7 de 08 de 2015, Topic Paper Process Documentation. Escrito para 'Making Connections A Neighbourhood Transformation FamilyDevelopmenteInitiative, Pagina 3.

Informáticas, S. C. (2012). Modeladodeprocesoscon IDEF en la metodología RUP. La Habana: Serie Científica de la Universidad de las Ciencias Informáticas, No. 2, Vol. 5 . Obtenido de http://publicaciones. uci.cu/index.php/SC

Métodos, Recuperado el 8 de 07 de 2013. Guía Técnica para Documentar Procedimientos. Jalisco, México.

Moreira, G. C. (2009). Diseño y propuesta de un modelo de gestión por procesos para la empresa licorera Lovisone. Ecuador. implementación propuesta con el fin de obtener una mejora continua general y sustentable de los procesos.
Público, D. G. (2010). Ministerio de Economía y Finanzas. Recuperado el 06 de 06 de 2015, de Instructivo para la Formulación de Indicadores de Desempeño. Obtenido de www.mef.gob.pe/ index.php?option=com_content\&view=article\&i $\mathrm{d}=2403$ \& Itemid=101582\&lang=es

Schouten, T. (2007). Process Recomendation in EMPOWERS. EMPOWERS Working Paper No. 8. Obtenido de http://www.empowers.info

Servicios, M. d. (s.f.). Manual metodológico para el análisis y documentación de procesos. Recuperado el 13 de 06 de 2015, de http://pmcg.minplan. gov.ar/html/publicaciones/manual/Manual_ MetAnaDocPro.pdf

Tributaria, S. N. (2011). Accedidoel5de08de2015, Guia de Referencia para el Usode IDEF0 . Peru. Obtenido de Guía de referencia para el Uso del IDEF0. 